06,11

\title{
Кинетика процесса поляризации в прозрачной керамике $\mathrm{Pb}\left(\mathrm{Mg}_{1 / 3} \mathrm{Nb}_{2 / 3}\right) \mathrm{O}_{3}-23 \mathrm{~Pb}\left(\mathrm{Zr}_{0.53} \mathrm{Ti}_{0.47}\right) \mathrm{O}_{3}$
}

\author{
(С) Л.С. Камзина ${ }^{1}$, Л.А. Кулакова ${ }^{1}$, G. $\mathrm{Li}^{2}$ \\ ${ }^{1}$ Физико-технический институт им. А.Ф. Иофрфе РАН, \\ Санкт-Петербург, Россия \\ ${ }^{2}$ Shanghai Institute of Ceramics, Chinese Academy of Sciences, \\ Shanghai, PR China \\ E-mail: Kamzin@mail.ioffe.ru
}

(Поступила в Редакцию 9 июля 2018 г.)

Исследована кинетика процесса поляризации в прозрачной сегнетокерамике $\mathrm{Pb}\left(\mathrm{Mg}_{1 / 3} \mathrm{Nb}_{2 / 3}\right) \mathrm{O}_{3}-$ $23 \mathrm{~Pb}\left(\mathrm{Zr}_{0.53} \mathrm{Ti}_{0.47}\right) \mathrm{O}_{3}$ в электрических полях $0<E<6 \mathrm{kV} / \mathrm{cm}$. При комнатной температуре проведены измерения оптического пропускания, диэлектрических и акустических свойств. Обнаружено, что даже в полях, меньших коэрцитивного, происходит мгновенное изменение диэлектрических и акустических свойств, связанное с возникновением частично упорядоченной сегнетоэлектрической фазы. Показано, что поляризованная фаза, возникшая в электрическом поле, не является полностью стабильной.

DOI: 10.21883/FTT.2019.01.46900.199

\section{1. Введение}

Свинцово-содержащие твердые растворы со структурой перовскита, состоящие из нормального сегнетоэлектрика и релаксора, интересны как с фундаментальной, так и с практической точки зрения. Составы, расположенные на морфотропной фазовой границе (МФГ), обладают превосходными диэлектрическими, пьезоэлектрическими и электромеханическими свойствами, что очень важно для практических применений. Монокристаллические релаксорные твердые растворы, такие как, $\mathrm{Pb}\left(\mathrm{Zn}_{1 / 3} \mathrm{Nb}_{2 / 3}\right) \mathrm{O}_{3}-\mathrm{PbTiO}_{3}$ (PZN-PT), $\quad \mathrm{Pb}\left(\mathrm{Mg}_{1 / 3} \mathrm{Nb}_{2 / 3}\right) \mathrm{O}_{3}-\mathrm{PbTiO}_{3} \quad$ (PMN-PT) и $\mathrm{Pb}\left(\mathrm{In}_{1 / 2} \mathrm{Nb}_{1 / 2}\right) \mathrm{O}_{3}-\mathrm{Pb}\left(\mathrm{Mg}_{1 / 3} \mathrm{Nb}_{2 / 3}\right) \mathrm{O}_{3}-\mathrm{PbTiO}_{3}$ (PIN$\mathrm{PMN}-\mathrm{PT})$, имеют большие величины пьезоэлектрического коэффициента $\left(d_{33} \sim 2000 \mathrm{pC} / \mathrm{N}\right)$ и коэффициента электромеханической связи $\left(k_{33} \sim 90 \%\right)$, что важно для их использования в медицинских ультразвуковых преобразователях и актюаторах. Однако, относительно низкие температуры Кюри и морфотропного фазового перехода (МФП) $\left(T_{c} \sim 400-470 \mathrm{~K}, T_{r t} \sim 330-370 \mathrm{~K}\right)$ могут ограничивать их применение в преобразователях, в которых требуется температурная стабильность.

Среди многочисленных твердых растворов подобного типа, интенсивно изучаемых в последнее время, цирконат-титанат свинца $\left(\mathrm{Pb}\left(\mathrm{Zr}_{1-x} \mathrm{Ti}_{x}\right) \mathrm{O}_{3}\right.$ или PZT) и магнониобат свинца (PMN) занимают особое место, так как каждый из компонент этого соединения является многообещающим кандидатом для применения в электронных и микроэлектронных устройствах $[1,2]$. Пьезо- и пироэлектрические свойства этих материалов представляют огромный интерес для создания эффективных и конкурентоспособных устройств, работающих в различных условиях. На PZT исследователи обратили пристальное внимание еще в 50 го- ды из-за их привлекательных свойств [3,4]. Наилучшими свойствами обладали составы PZT с соотношением $\mathrm{Zr} / \mathrm{Ti} 53 / 47$, расположенные вблизи МФГ между тетрагональной и ромбоэдрической фазами. В них наблюдался большой пьезоэлектрический эффект, высокий коэффициент электромеханической связи, который больше, чем в $\mathrm{PMN}$, и большая диэлектрическая проницаемость. Однако недостатками PZT являются высокие диэлектрические потери и большое коэрцитивное поле. Это делает PZT неудобным объектом для применений.

PMN является релаксором, имеет низкую температуру синтеза, высокую диэлектрическую проницаемость, большую деформацию и широкий максимум диэлектрической проницаемости. Кроме того, PMN имеет низкие потери и узкую петлю гистерезиса. Твердые растворы PMN-PZT обладают лучшими пьезоэлектрическими и диэлектрическими свойствами, чем отдельно взятые PMN и PZT [5]. Выбор таких материалов обусловлен, кроме хороших пьезоэлектрических свойств, еще несколькими причинами. Во-первых, одна из краевых компонент твердого раствора (PMN) является модельным и хорошо изученным релаксором, а PZT - относится к сегнетоэлектрикам, претерпевающим обычный сегнетоэлектрический фазовый переход. Во-вторых, твердые растворы PMN-PZT можно получать по хорошо отработанной керамической технологии в виде массивных образцов с воспроизводимыми свойствами и необходимого для исследований состава. В-третьих, оба состава из выбранной системы хорошо изучены. Последнее обстоятельство облегчает интерпретацию полученных ранее результатов [6].

В работах $[7,8]$ изучались диэлектрические свойства керамик $\mathrm{PMN}-x \mathrm{PZT} \mathrm{c}$ разной величиной $x$. Было показано, что МФГ между тетрагональной и псевдо- 
кубической (ромбоэдрической) фазами лежит вблизи $x \sim 0.4-0.5$. Переходная область между релаксорным и нормальным сегнетоэлектрическим поведением наблюдается в составах, расположенных в композиционной области $0.40<x<0.50$. При комнатной температуре в этой переходной области наблюдается сосуществование псевдокубической и ромбоэдрической фаз. Обычно фазовый переход между ромбоэдрической и тетрагональной фазами (МФП) происходит не сразу, а через промежуточную моноклинную или орторомбическую фазы. Это подтверждено многочисленными исследованиями на бинарных и тройных системах типа $\mathrm{PMN}-x \mathrm{PT}$, PIN-PMN-PT и ряда других [9-11]. Однако ситуация в соединениях PMN- $x$ PZT, как показано в работе [12], существенно отличается от предыдущей. Никаких низкосимметричных фаз (моноклинной или орторомбической) не обнаружено при МФП, а в переходной области наблюдается переход из ромбоэдрической фазы с ближним порядком в ромбоэдрическую фазу с дальним порядком. Этот переход не требует вращения поляризации, как в случае промежуточной моноклинной или орторомбической фазы. Отсутствие промежуточной фазы, и при этом наличие досточно большого пьезоэффекта, делают соединения PMN-xPZT особенно привлекательными для исследователей.

В наших работах [13-15], на впервые приготовленной нами прозрачной керамике $\mathrm{Pb}\left(\mathrm{Mg}_{1 / 3} \mathrm{Nb}_{2 / 3}\right) \mathrm{O}_{3}-$ $x \mathrm{~Pb}\left(\mathrm{Zr}_{0.53} \mathrm{Ti}_{0.47}\right) \mathrm{O}_{3} \quad(\mathrm{PMN}-x \mathrm{PZT})$ составов с $x=23$ и $33 \%$ при высоких температурах $(\sim 340 \mathrm{~K})$ было обнаружено наивысшее значение квадратичных электрооптических (ЭО) коэффициентов по сравнению с их значениями в других известных ЭО-материалах.

Напомним, что необычные свойства релаксорных материалов связаны с присутствием пространственно ориентированных сегнетоэлектрических наноразмерных областей ромбоэдрической симметрии (полярные нанообласти PNR), которые возникают при высокой температуре (температура Бернса) и замерзают при охлаждении при некоторой температуре (температура замерзания, или температура Фогеля-Фулчера $T_{f}$ ). В замороженном релаксорном состоянии PNR приобретают слабую кинетику, но не образуют дальнего порядка. Свойства системы в таком неравновесном состоянии должны зависеть от времени. В электрическом поле происходит трансформация стекольного состояния в фазу с дальним порядком. Немногочисленные опубликованные работы по кинетике такого перехода были посвящены только отдельным кристаллическим соединениям типа $\mathrm{PMN}-x$ PT и PIN-PMN- $x$ PT [16-19]. Однако динамика такого перехода в системе $\mathrm{PMN}-x \mathrm{PZT}$ вообще не изучалась.

В настоящей работе изучена кинетика процесса поляризации в прозрачной сегнетокерамике 77PMN-23PZT. Измерены временные зависимости изменения диэлектрической проницаемости и упругих свойств (скорости и затухания звука), а также оптического пропускания в различных электрических полях.

\section{2. Исследуемые образцы и методика эксперимента}

Прозрачная керамика PMN-23PZT с соотношением $\mathrm{Ti}: Z r$ равным 53:47, приготовлялась с использованием необычного двухступенчатого метода спекания, описанного в нашей работе [13]. Для того чтобы увеличить вероятность образования перовскитовой фазы и уменьшить присутствие пирохлорной фазы при приготовлении керамики PMN-23PZT, вместо цирконата свинца использовался цирконат-титанат свинца [13]. Полученные образцы были высокого оптического качества. Для оптических измерений спеченные образцы полировались до толщины $0.5 \mathrm{~mm}$. Пропускание образцов при комнатной температуре в области 600-2100 nm составляло $\sim 65 \%$. Температура Кюри $T_{c} \sim 340 \mathrm{~K}$, температура Фогеля-Фулчера $T_{v f} \sim 315-320 \mathrm{~K}$.

Измеритель иммитанса Е7-15 использовался для диэлектрических измерений. Для оптических измерений

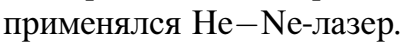

Данные об изменении упругих постоянных $\Delta C / C$ получены из измерений скорости $(V)$ продольных ультразвуковых волн, распространяющихся вдоль электрического поля. Звук с частотой $20 \mathrm{MHz}$ возбуждался с помощью резонансного пьезопреобразователя, изготовленного из поляризованной пьезокерамики ЦТС (PZT). Для измерения скорости звука использовалась эхо-импульсная методика, которая была подробно описана ранее в нашей работе [20].

Все измерения проводились при комнатной температуре в постоянных электрических полях напряженностью от 0 до $6 \mathrm{kV} / \mathrm{cm}$. При комнатной температуре керамика PMN-23PZT находится в основном в неэргодической релаксорной фазе с небольшой долей нанообластей ромбоэдрической симметрии.

Перед каждым измерением образцы отжигались в течение $30 \mathrm{~min}$ при температуре $\sim 600 \mathrm{~K}$, чтобы устранить в образцах эффекты памяти, связанные с приложением электрического поля. После отжига образцы охлаждались без поля до комнатной температуры, при которой к ним прикладывалось постоянное электрическое поле. После приложения электрического поля начинался отсчет времени и осуществлялась регистрация изменения диэлектрической проницаемости, скорости и поглощения звука. Измерения проводились непосредственно после отжига образцов.

\section{3. Экспериментальные результаты и их обсуждение. Диэлектрические и оптические свойства}

На рис. 1 (кривые 1-7) представлены временные зависимости изменений диэлектрической проницаемости $\left(\varepsilon / \varepsilon_{0}\right)$ в разных электрических полях. Видно, что после включения электрического поля величина $\varepsilon / \varepsilon_{0}$ уменьшается со временем во всех электрических полях. Незначительное изменение $\varepsilon$ в отсутствие электрического поля 
(кривая 1) связано с естественным старением керамики. Поля до $1 \mathrm{kV} / \mathrm{cm}$ также не приводят к существенным изменениям $\varepsilon$ (кривая 2). Более быстрое и заметное изменение $\varepsilon$ наблюдается в электрическом поле, превышающем $1 \mathrm{kV} / \mathrm{cm}$ (кривая 3). С дальнейшим увеличением электрического поля за короткий промежуток времени (кривые 4-7) происходит резкое уменьшение $\varepsilon$, свидетельствующее об увеличении доли сегнетоэлектрической фазы. Можно предположить, что столь сильное уменьшение величины $\varepsilon$ связано с уменьшением числа межфазных границ, укрупнением в электрическом поле нанодоменов ромбоэдрической фазы и переходом части объема образца в макродоменное состояние.

Выключение электрического поля приводит к скачкообразному возрастанию величины $\varepsilon$ (рис. 2, кривые 1-3): однако, значение ее меньше, чем до приложения поля. Это может быть связано с возвращением

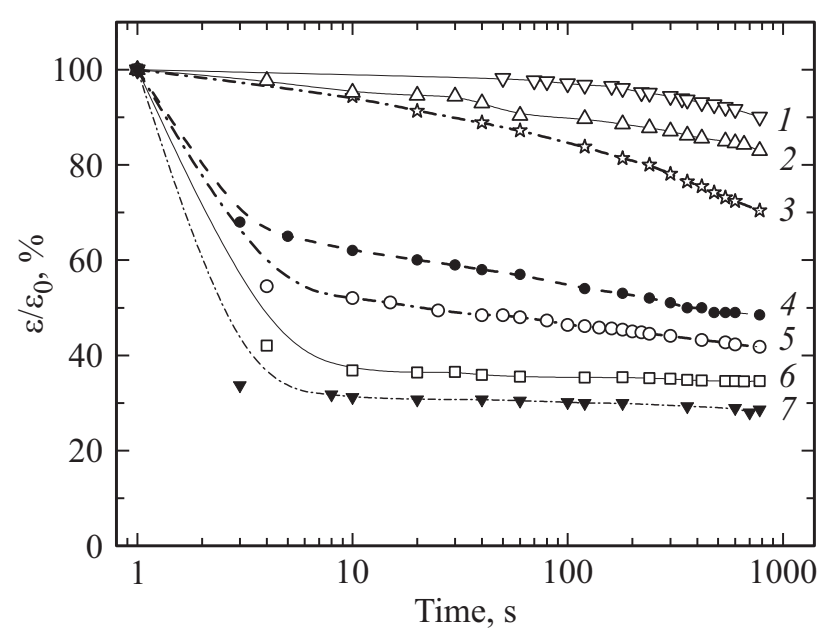

Рис. 1. Относительные изменения диэлектрической проницаемости $\left(\varepsilon / \varepsilon_{0}\right)\left(\varepsilon_{0}-\right.$ величина в нулевом поле) со временем в разных электрических полях $E, \mathrm{kV} / \mathrm{cm}: 1-0,2-0.8,3-$ $1.1,4-1.5,5-2,6-4,7-6$.

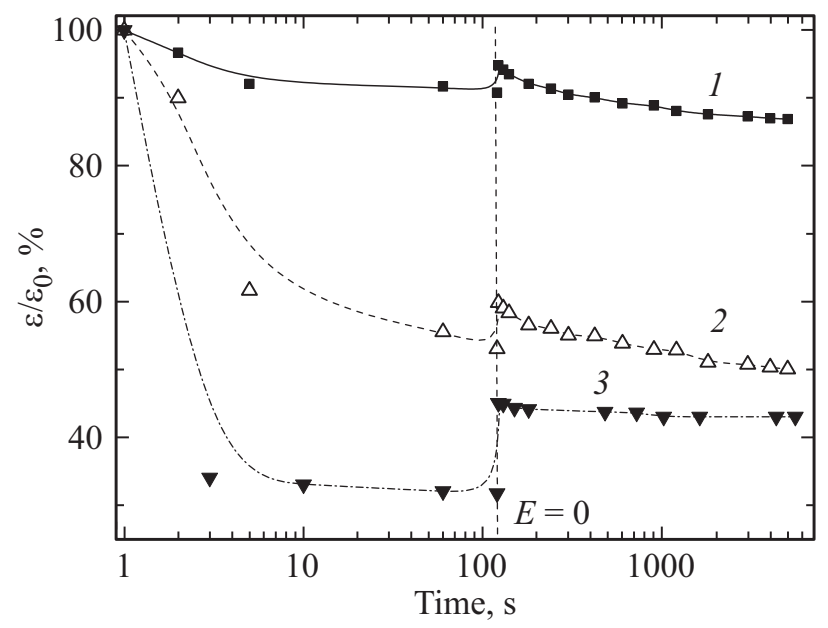

Рис. 2. Временные зависимости диэлектрической проницаемости до и после выключения электрического поля разной напряженности $E, \mathrm{kV} / \mathrm{cm}: 1-1,2-2,3-6$.

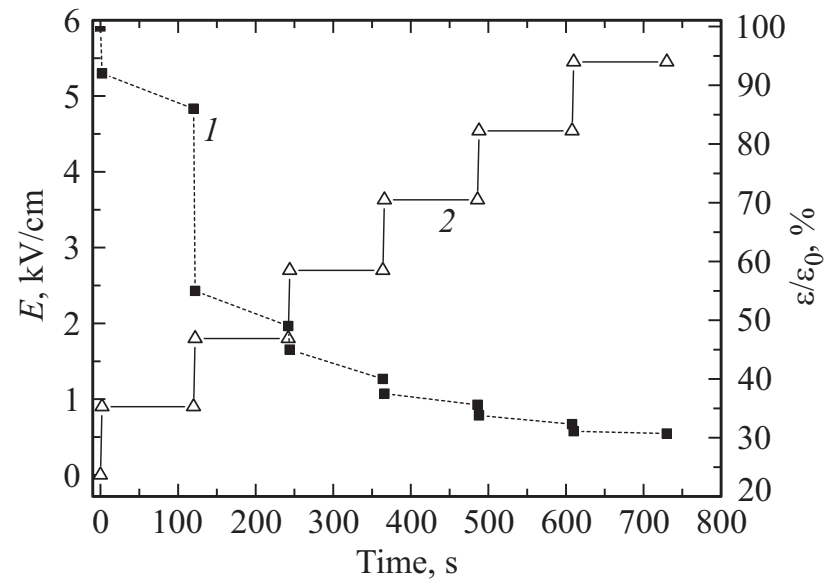

Рис. 3. Изменение величины $\varepsilon / \varepsilon_{0}$ (кривая 1) со временем при увеличении электрического поля (кривая 2). Каждое поле прикладывалось в течение двух $\min$.

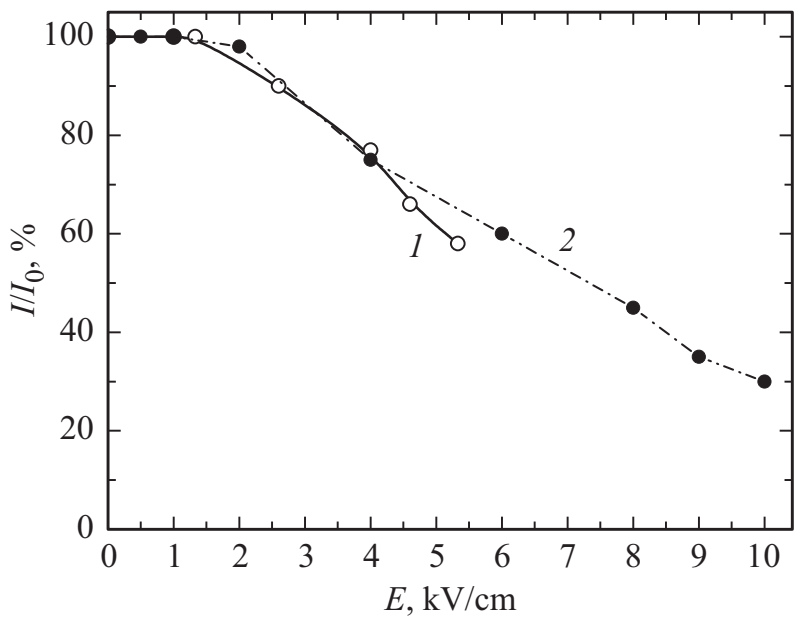

Рис. 4. Зависимость относительного изменения интенсивности оптического пропускания $\left(I / I_{0}\right)$ от напряженности электрического поля $\left(I_{0}-\right.$ первоначальное значение оптического пропускания в отсутствие электрического поля): 1 - наши измерения, 2 - данные, взятые из работы [15].

не всего объема кристалла, а только определенной доли заполяризованных областей в релаксорное состояние. В полях, существенно меньших коэрцитивного $\left(E_{c} \sim 6.5 \mathrm{kV} / \mathrm{cm}\right)$ после скачка (кривые 1,2$)$ наблюдается небольшое уменьшение $\varepsilon / \varepsilon_{0}$, что может быть следствием проявления процессов естественного старения. Возникшая же в полях, близких к $E_{c}$, частично упорядоченная сегнетоэлектрическая структура остается практически стабильной после выключения поля (рис. 2, кривая 3) во временном интервале, используемом в данной работе (до двух h). Следовательно, фаза, возникшая в электрическом поле, связана, скорее всего, с переходом части объема образца в сегнетоэлектрическую ромбоэдрическую фазу.

Наиболее наглядно процесс лавинообразного изменения $\varepsilon$ иллюстрируется рис. 3 , на котором представлено 
изменение величины $\varepsilon / \varepsilon_{0}$ со временем при изменении электрического поля. Каждое поле прикладывалось последовательно и выдерживалось в течение двух min. Измерения диэлектрической проницаемости проводились в начальный момент приложения поля и спустя две $\mathrm{min}$. Из кривой 1 видно, что приложение поля до $1 \mathrm{kV} / \mathrm{cm}$ в течение двух $\min$ изменяет величину $\varepsilon / \varepsilon_{0}$ всего на $5 \%$, в то время как приложение поля $\sim 2 \mathrm{kV} / \mathrm{cm}$ в течение того же времени почти на $30 \%$, причем изменения происходят практически мгновенно после приложения поля. Дальнейшее увеличение поля до $6 \mathrm{kV} / \mathrm{cm}$ приводит к насыщению изменения величины $\varepsilon / \varepsilon_{0}$.

Результаты измерений оптического пропускания в электрических полях не противоречат диэлектрическим измерениям. Из рис. 4 (кривая 1) видно, что интенсивность оптического пропускания уменьшается с увеличением напряженности электрического поля. Аналогичная зависимость наблюдалась для прозрачной керамики того же состава авторами работы [15] (кривая 2). Видно хорошее совпадение обеих кривых. Уменьшение пропускания обусловлено ростом электрически индуцированных полярных областей или доменов. Возникновение в поле частично упорядоченной сегнетоэлектрической структуры или доменов больших размеров может приводить, соответственно, к большим доменным стенкам, которые рассеивают падающий свет. Из рис. 3 и 4 видно, что характер поведения диэлектрического и оптического откликов при изменении величины постоянного электрического поля идентичен. Это еще раз подтверждает преобладающий вклад доменов в пропускание прозрачной керамики.

\section{4. Акустические свойства}

На рис. 5 приведена кинетика изменения упругой постоянной исследуемой керамики в фиксированном электрическом поле $(E=4 \mathrm{kV} / \mathrm{cm})$. Из данных этого рисунка следует, что приложение поля приводит сначала к скачкообразному увеличению жесткости, которое затем сменяется слабым нарастанием упругой постоянной в присутствии поля. Выключение поля приводит к столь же резкому уменышению упругости. Однако, возвращения к исходному значению скорости звука, так же, как в случае диэлектрических измерений (рис. 2), не наблюдается. Со временем эта величина уменьшается незначительно (рис. 5,6).

Изменение упругих свойств как затухания, так и скорости звука в электрическом поле, как видно из рис. 6 носит пороговый характер: скачкообразное изменение их возникает в условиях превышения величины поля над его пороговой величиной $E_{\text {пор }} \approx 1.5-2 \mathrm{kV} / \mathrm{cm}$. Дальнейшее относительно плавное увеличение электрического поля приводит к достаточно плавному возрастанию как скорости, так и затухания звука (рис. 6). Существенное замедление изменения упругих свойств происходит в поле $\approx 6 \mathrm{kV} / \mathrm{cm}$, как видно из рис. 6,7 . Выключение поля приводит к резкому уменьшению как скорости, так и затухания звука, величина которых со временем после выключения поля медленно уменышается.

Наиболее ярко влияние процессов поляризации на упругие свойства можно проследить на рис. 7, где представлены временные зависимости упругой постоянной при увеличении электрического поля с выдержкой в каждом поле в течение 2-х минут. Из рисунка видно, что каждое увеличение поля при переключении приводит к скачкообразному изменению упругой постоянной, сменяющееся дальнейшим медленным нарастанием ее в течение последующих двух min. Выключение поля приводит, как уже отмечалось выше, к скачкообразному уменьшению упругой постоянной (скорости звука) до величины, составляющей $\sim 45 \%$ от значения в максимальном поле $(6 \mathrm{kV} / \mathrm{cm})$ с последующим медленным уменьшением на несколько процентов.

Результаты акустических измерений рис. 7 качественно совпадают с диэлектрическими данными рис. 3, измеренными в тех же условиях приложения поля. В обоих случаях наблюдается пороговый характер зависимости,

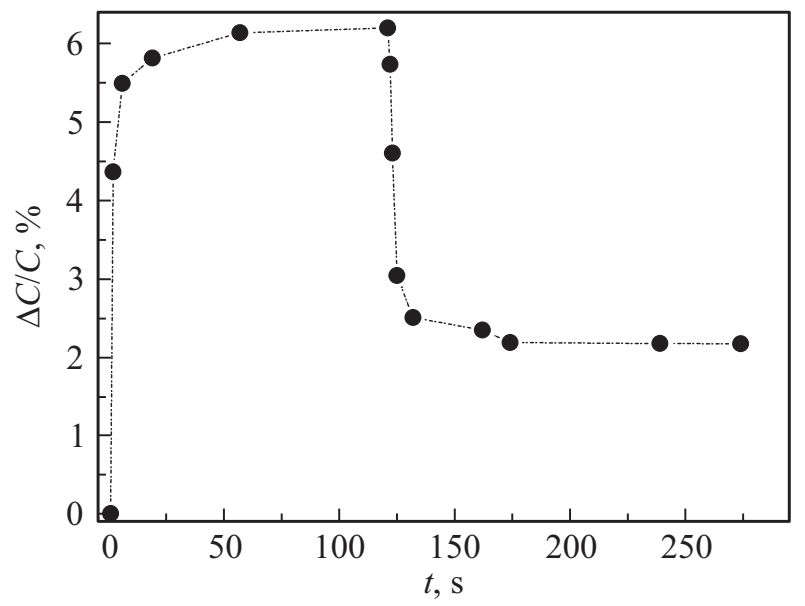

Рис. 5. Временная зависимость изменения упругой постоянной в фиксированном электрическом поле $(E=4 \mathrm{kV} / \mathrm{cm})$.

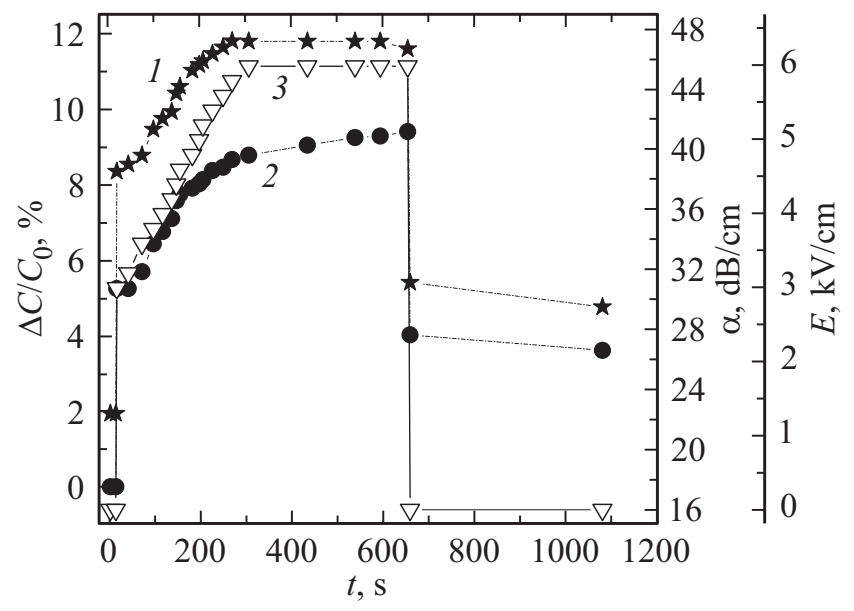

Рис. 6. Временная зависимость затухания (кривая 1) и упругой постоянной (кривая 2) при плавном увеличении электрического поля (кривая 3). 


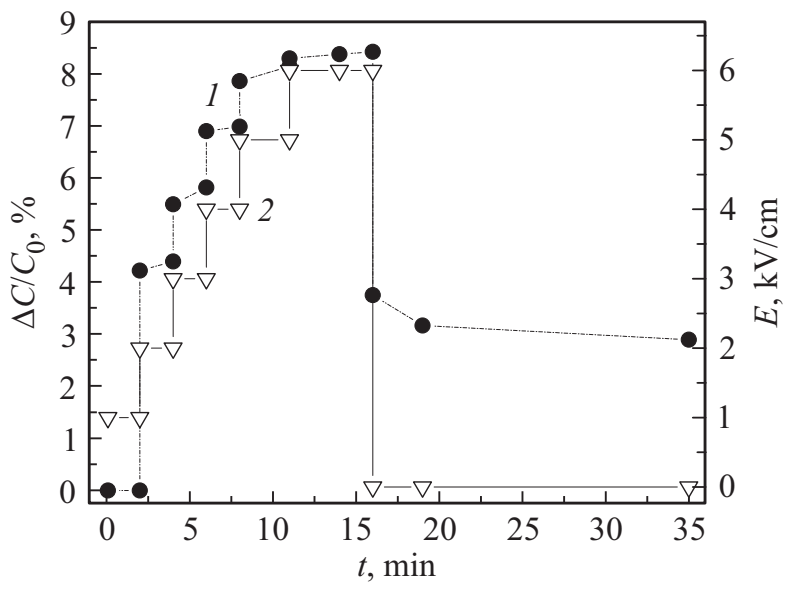

Рис. 7. Зависимость от времени упругой постоянной (кривая 1) при увеличении электрического поля (кривая 2). Каждое поле прикладывалось в течение двух min.

причем основные изменения происходят при приложении поля $\sim 1.5-2 \mathrm{kV} / \mathrm{cm}$.

Из комплекса представленных данных следует, что в электрическом поле происходит поляризационное упорядочение лишь отдельных частей объема образца. В результате наблюдается увеличение жесткости решетки и роста затухания звука за счет процессов рассеяния, аналогичным образом проявляющихся в изменениях оптического пропускания. Такое упорядочение не является стабильным, поскольку сохраняется лишь частично после выключения поля.

Подтверждением того, что даже в полях $\sim 6 \mathrm{kV} / \mathrm{cm}$ происходит лишь частичное поляризационное упорядочение и сегнетоэлектрический фазовый переход происходит не во всем объеме образца, служит тот факт, что изменение упругих свойств не зависит от знака приложения поля. Действительно, нами обнаружено, что приложение поля разной полярности приводит к одинаковому изменению акустических свойств, а именно, - к возрастанию скорости звука. Если бы имел место фазовый переход из неэргодической релаксорной фазы в сегнетоэлектрическую фазу во всем объеме образца, то возникал бы пьезоэлектрический эффект, и тогда изменение знака поля должно приводить к соответствующему знакопеременному изменению скорости звука. Это отличает исследуемую нами керамику при приложении полей до $\sim 6 \mathrm{kV} / \mathrm{cm}$ от заполяризованой в больших полях пьезокерамики ЦТС, в которой мы наблюдали соответствующее увеличение и уменьшение скорости звука в полях противоположного знака.

\section{5. Выводы}

1. Обнаружено, что даже в электрических полях меньших коэрцитивного поля, происходит практически мгновенное изменение диэлектрических и акустических свойств, связанное с фазовым переходом из ромбоэдрической фазы с ближним порядком в ромбоэдрическую фазу с дальним порядком. Лавинообразное изменение основных параметров определяет пороговый характер кинетики процессов поляризации.

2. В электрическом поле происходит поляризационное упорядочение лишь отдельных частей объема образца. В результате наблюдается увеличение жесткости решетки и роста затухания звука за счет процессов рассеяния, аналогичным образом проявляющихся в изменениях оптического пропускания.

3. Показано, что поляризованная фаза, возникшая в электрическом поле, не является полностью стабильной. Об этом свидетельствует скачкообразное увеличение диэлектрической постоянной и уменьшение скорости и затухания звука после выключения поля. Измеряемые величины не возвращаются к исходным до приложения поля величинам. Это подтверждает факт не полной, а только частичной, поляризации образца в полях, меньших коэрцитивного.

4. С увеличением поля увеличивается доля поляризованного объема образца, перешедшего в ромбоэдрическую фазу, о чем свидетельствует наблюдаемое насыщение изменения акустических и диэлектрических параметров в полях ( $6 \mathrm{kV} / \mathrm{cm})$, близких к коэрцитивному.

\section{Список литературы}

[1] L.E. Cross. Mater. Chem. Phys. 43, 108 (1996).

[2] G.H. Haertling. J. Am. Ceram. Soc. 82, 797 (1999).

[3] R. Shannigrahi, R.N.P. Choudhary, H.N. Acharya. Mater. Lett. 39, 318, (1999).

[4] S.K.S. Parashar, R.N.P. Choudhary, B.S. Murty. J. Appl. Phys. 94, 6091 (2003).

[5] R. Yimnirun, S. Ananta, E. Meechoowas, S. Wonsaenmai. J. Phys. 36, 1615 (2003)

[6] L.X. He, M. Gao, C.E. Li, W.M. Zhu, H.X. Yan. J. Eur. Ceram. Soc. 21, 703 (2001).

[7] R. Yimnirun, S. Ananta, P. Laoratakul, S. Songklanakarin. J. Sci. Technol. 26, 529 (2004).

[8] Shujun Zhang, Sung-Min Lee, Dong-Ho Kim, Ho-Yong Lee, Thomas R. Shrout. J. Am. Ceram. Soc. 90, 3859 (2007).

[9] S.-E. Park, T.R. Shrout. J. Appl. Phys. 82, 1804 (1997).

[10] J. Kuwata, K. Uchino, S. Nomura. Ferroelectrics 37, 579 (1981).

[11] D. Vieland, J. Powers. J. Appl. Phys. 89, 1820 (2001).

[12] G. Singh, V.S. Tiwari. J. Appl. Phys. 101, 014115 (2007).

[13] W. Ruan, G.R. Li, J.T. Zeng, L.S. Kamzina, H.R. Zeng, L.Y. Zheng, A.L. Ding. J. Am. Ceram. Soc. 95, 2103 (2012).

[14] Л.С. Камзина, Wei Ruan, Guorong Li, Jiangtao Zeng. ФТT 54, 1899 (2012).

[15] W. Zhao, W. Ruan, J. Zeng, L. Huang, K. Zhao, L. Zheng, H. Zeng, Y. Zhou, H. Yang, X. Ruan, Guorong Li. Appl. Phys. Lett. 104, 062907 (2014).

[16] E.V. Colla, E.Y. Koroleva, N.M. Okuneva, S.B. Vakhrushev. Phys. Rev. Lett. 74, 1681 (1995).

[17] E.V. Colla, N. Jurik, Y. Liu, M.E.X. Delgado, M.B. Weissman, D.D. Vieland, Z.-G. Ye. J. Appl. Phys. 113, 184104 (2013).

[18] Л.С. Камзина, Л.А. Кулакова. ФТТ 59, 290 (2017).

[19] V. Koval, C. Alemany, J. Briancin, H. Brunckova, K. Saksl. J. Eur. Ceram. Soc. 23, 1157 (2003).

[20] Л.С. Камзина, Л.А. Кулакова, Н. Luo. ФТТ 56, 9, 1809 (2014).

Редактор Т.Н. Василевская 